\title{
Delayed Ureteral Fistula After Laparoscopic Lateral Lymph Node Dissection for Rectal Cancer: A Case Report and Literature Review
}

ying yang ( $\sim$ yangyingpwkjca@126.com )

Sichuan University West China Hospital

\section{Xiang-Bing Deng}

Sichuan University West China Hospital

Ming-Tian Wei

Sichuan University West China Hospital

Wen-Jian Meng

Sichuan University West China Hospital

Zi-Qiang Wang

Sichuan University West China Hospital

\section{Research Article}

Keywords: Lateral lymph node metastasis, Lateral lymph node dissection, Ureteral injury, Neoadjuvant chemoradiotherapy,

Posted Date: October 18th, 2021

DOl: https://doi.org/10.21203/rs.3.rs-958862/v1

License: (c) (1) This work is licensed under a Creative Commons Attribution 4.0 International License. Read Full License 


\section{Abstract}

Background: Ureteral injury is the most common urological complication of pelvic surgery. Lateral lymph node dissection (LLND) is a technically demanding procedure by laparoscopy after neoadjuvant chemoradiotherapy (nCRT), which may increase the risk of ureteral injury because the ureter and hypogastric fascia must be dissected from the vesicohypogastric fascia to preserve them.

Case presentation: Here, we present a case of delayed ureteral leakage occurring in a 63-year-old male patient who underwent laparoscopic abdominoperineal resection (APR) with right LLND after nCRT. Ureteral reimplantation (vesicoureteric anastomosis) was performed in this patient due to the failure of the placement of the double $\mathrm{J}$ stent.

Conclusions: Our case shows that ureteral leakage may occur in the pelvic segment of the ureter in the patient who underwent laparoscopic LLND, especially received preoperative radiotherapy or chemoradiotherapy.

\section{Background}

For locally advanced rectal cancer with lateral lymph node metastasis (LLNM), total mesorectal resection (TME) plus neoadjuvant chemoradiotherapy (nCRT) are often used internationally; in Japan, TME plus lateral lymph node dissection (LLND) is the standard treatment[1], while in China, TME combined with selective LLND after nCRT are recommended[2]. latrogenic ureteral injury is a rare complication in colorectal surgery $(0.15 \%-1.9 \%)$ [3], but is associated with serious consequences. Intraoperative identification of ureteral injury enables timely management of the damaged ureter, but unfortunately, the likelihood of intraoperative detection of ureteral injury is low[4]. Here, we report a case of a rectal cancer patient with delayed ureteral fistula after nCRT following with laparoscopic LLND. In this report, we describe the clinical procedures for the complication in detail and review the literatures in order to improve the understanding of delayed ureteral fistula after LLND for rectal cancer, avoid its missed diagnosis and provide guidance for its clinical treatment.

\section{Case Presentation}

A 63-year-old man was admitted to local hospital with chief complaint of defecation difficulty for one week. Colonoscopy after enema suggests rectal neoplasm and pathological examination confirmed rectal adenocarcinoma. One day after colonoscopy, sigmoid colostomy was emergently performed in this patient because of colonic perforation. The patient then came to our hospital to consult for further treatment. Colonoscopy in our hospital showed that ulcerative neoplasm located $8 \mathrm{~cm}$ from the anal verge invaded about two-thirds of the circumferential intestinal wall and pathological examination showed mucinous adenocarcinoma with mucinous component and few signet-ring cells. Clinically suspected LLNM on the right side was considered based on contrast-enhanced computed tomography (CT) and magnetic resonance imaging (MRI) (Figure 1), then he was planned to administer nCRT. After 
two courses chemotherapy of XELOX (Oxaliplatin infusion + capecitabine taken orally) and radiotherapy with 50.4 Gy (28 fractions) accompanied by oral capecitabine in total, the sizes of both the primary tumor and lymph nodes decreased (partial response: PR) (Figure 2).

Curative radical resection was performed 6-8 weeks after the completion of radiotherapy. After the multidisciplinary team evaluation, laparoscopic abdominoperineal resection (APR) with right LLND was suggested. During the operation, an extended radical surgery for rectal cancer was performed to remove of the suspiciously invaded the right seminal vesicle gland and the right pelvic nerve. After completion of the TME procedure, LLND was performed according to our previously reported method[5]. Briefly, LLND began by isolating the ureter, hypogastric nerve, and pelvic nerves from the pelvic sidewall. Thereafter, the dissection of lymph nodes was initiated in the area around the common iliac artery, around the common iliac artery, internal iliac artery, and obturator space. Because LLNM most frequently occurs around the distal internal iliac vessels, LLND with the combined vesical artery resection may simplify the procedure and bring on more secure en bloc dissection of metastatic lateral lymph node adjacent to the vessels. Thus, for this case, the anterior trunk of right internal iliac artery was ligated, leaving only the superior gluteal artery during LLND. The patient was discharged on 7th after surgery without immediate postoperative complications and voiding dysfunction. Next, he underwent four cycles chemotherapy of XELOX and no recurrence was found during regular follow-up after surgery.

However, one month after the second surgery, the patient presented to our outpatient clinic with chief complaints of frequent micturition, urgency of urination and intermittent fever. These symptoms improved after the treatment of antibiotics but recurred since then. Five months after the second surgery, the patient was readmitted to our hospital with chief complaints of fluid drained from the perineal wound. Physical examination showed that perineum was distended with tenderness and no signs of peritoneal irritation were observed. And clear fluid drained from a fistula about $2 \mathrm{~mm}$ in size located at the upper part of perineal wound. Contrast-enhanced CT revealed there was encapsulated effusion in pelvic cavity, which involved bladder and right lower ureter. Moreover, there was hydronephrosis in the upper part of right ureter and renal pelvis, right lower ureteral stricture was found and the perfusion of right kidney was decreased. Due to apparent pelvic effusion, fistula enlargement and placement of drainage tube through the perineal fistula were performed. The amount of fluid reached about $850 \mathrm{ml}$ on the first day after drainage tube placement. Biochemical results of drainage fluid showed creatinine $918 \mathrm{umol} / \mathrm{L}$, uric acid $1092 \mathrm{umol} / \mathrm{L}$, urea $41.6 \mathrm{mmol} / \mathrm{L}$, serum cystatin $C$ determination $0.23 \mathrm{mg} / \mathrm{L}$, estimated glomerular filtration rate $4.78 \mathrm{ml} / \mathrm{min} / 1.73 \mathrm{~m}^{2}$. Moreover, computed tomography urography (CTU) showed that the fistula between the right pelvic segment of the ureter and the encapsulated effusion area was formed, the right pelvic segment of ureter in the surgical area was unclear and dilatation of ureter was found above that part of ureter and the perfusion of the right kidney was decreased (Figure $3 \mathrm{~A}$ ). These results suggested the possibility of a right pelvic ureteral fistula. Therefore, cystoscopic double-J ureteral stent insertion was performed. The postoperative course was uneventful, and the patient was discharged on postoperative day 4 . The patient had been carrying drainage tubes and double-J ureteral stent since discharge, and no pelvic effusion or fever occurred. 
However, five months after the ureteral stent insertion, the pelvic drainage tube was accidentally removed by the patient, followed by massive pelvic effusion and persistent fever. Enhanced ureteral CT revealed formation of right pelvic ureteral fistula and pelvic encapsulated effusion (Figure 3B). After the failure of replacement of the drainage tube through perineum, a laparoscopic ureteral reimplantation (vesicoureteric anastomosis) was performed and a new ureteral stent was placed after the ureteral leakage was identified in the right lower ureter (Figure 4). During the follow-up period, enhanced ureteral CT showed no leakage of contrast medium (Figure 5) and no pelvic effusion and fever were found in this patient with normal urinary function.

\section{Discussion And Conclusions}

Pelvic surgery is a very common cause of iatrogenic ureteral injury, with the estimated incidence is $0.5-3 \%$ [6]. In rectal surgery, the incidence of iatrogenic ureteral injury is likely to increase due to the popularization of laparoscopic surgery and the technical difficulty of the surgical procedures in the implementation of LLND. To our knowledge, this is the first report on describing a delayed ureteral injury after nCRT combined with APR plus laparoscopic LLND for rectal cancer. A previous study suggests that laparoscopic surgery is likely to increase risk of ureteral injury compared with open surgery[7]. What's worse, ureteral injury caused by minimally invasive techniques such as laparoscopy is not easy to detect during surgery and is often considered only when symptoms of urinary tract infection occur after surgery[7]. In recent years, preoperative implementation of neoadjuvant radiotherapy has become the standard treatment modality for locally advanced rectal cancer. In these patients, dense adhesions and scar tissue of pelvic cavity make the boundary indistinct between the rectum and ureter, which makes the ureter more vulnerable to injury in surgery. In addition, radiation-induced fibrosis of ureteric mucosa leads to decreased ureteral compliance and subsequent increased wall tension, which may result in delayed postoperative ureteral fistula[8]. Gellrich et al have even reported that stenosis and necrosis of the lower ureter after radiotherapy in patients with cervical cancer[9]. And the patient's previous surgical history in this case also increased the technical difficulty of surgery. A review of the archived surgical video revealed no evidence of direct sharp of injury to urine. Except for above reasons, the possible causes of delayed ureteral leakage included the following points. When the APR plus laparoscopic LLND was performed, dissection and traction of ureter were needed. Therefore, the first possible cause of delayed ureteral leakage is excessive dissection of ureter. The blood supply of the ureter is derived from the branches of the blood vessels passing through the ureter. The small blood vessels feeding the ureter form a blood-rich vascular anastomosing network with each other in the outer membrane of the ureter. Excessive dissection of the ureter therefore leads to the loss of blood supply from periureteral vascular network, resulting in postoperative local ischemic necrosis of the ureter, thereby forming a ureteral fistula. The second possible cause of delayed ureteral leakage is excessive traction of ureter. This is similar to the speculation in a recent case report. Chikazawa et al. reported one patient with delayed ureteral leakage after laparoscopic hysterectomy for stage IB1 cervical adenocarcinoma[10]. They believed that delayed ureteral leakage was secondary to ischemia resulting from excessive traction of ureter using suspension tapes during surgery. Another possibility is the local damage to the ureter caused by energy 
devices such as monopolar or bipolar cautery. When dissecting the ureter, the heat generated by the electrotome may cause thermal damage to the ureter, but it is difficult to detect this damage during operation, which may lead to the stenosis and delayed fistula of ureter.

Ureteral fistula is often not diagnosed in the early stages because of the absence of some specific clinical manifestations, until the presence of upper urinary tract obstruction, urinary fistula formation or sepsis. CTU is the preferred method in patients with these above symptoms.

To avoid unnecessary damage to the ureter, an adequate understanding of ureteral anatomy and a meticulous surgical technique are first required. The risk of ureteral injury should be assessed preoperatively, and patients with locally advanced, locoregional recurrences and radiation therapy should be considered to be as high-risk cases of intraoperative ureteral injury. These factors, especially preoperatively radiotherapy, cause severe adhesion of the ureter with the peripheral tissues, which will make the ureter highly susceptible to injury during surgery. For these patients, pelvic CT, ultrasonography of the kidneys and urinary tract or intravenous pyelography should be routinely performed to fully understand the anatomy and course of the ureter. In addition, double-J ureteral stenting is recommended preoperatively for those patients to help identify the ureter during surgery and thus reduce the possibility of intraoperative damage to the ureter. In LLND, the location and course of the ureter should first be determined and the ureter should be pushed medially, but excessive traction of the ureter should be avoided during the surgery. Meanwhile during mobilization of the ureter, excessive dissection of the ureter should be avoided to preserve the peritoneum on the surface of the ureter. This will help to ensure the supply of blood to the ureter and thus reduce the risk of ureteral ischemia after surgery. In addition, the use of dissecting scissors may be considered to avoid thermal damage to the ureter by energy devices when dissecting the ureter.

In conclusion, there is a risk of delayed ureteral leakage in patients undergoing LLND after nCRT. The surgeon planning to perform the LLND (especially for patients with preoperative radiotherapy) should be familiar with the anatomy of the ureter, the risk factors of ureteral injury and the correct management of ureteral injury. In addition, the blood supply of the ureter, especially the pelvic segment of the ureter, should be protected during the LLND after radiotherapy, otherwise postoperative ischemic necrosis of ureteral may occur.

\section{Abbreviations}

LLND Lateral lymph node dissection

nCRT neoadjuvant chemoradiotherapy

APR abdominoperineal resection

LLNM lateral lymph node metastasis

TME total mesorectal resection 
CT computed tomography

MRI magnetic resonance imaging

CTU computed tomography urography

\section{Declarations}

- Ethics approval and consent to participate: Not applicable

- Consent for publication: The patient agrees to publish all statements and pictures of the case report and has signed the informed consent form.

- Availability of data and materials: Not applicable

- Competing interests: The authors declare that they have no competing interests

- Funding: No funding

- Author Contributions:

YY: data acquisition, data analysis and interpretation, manuscript drafting. XBD: data analysis and interpretation, manuscript drafting. MTW: data acquisition, data analysis and interpretation. WJM and ZQW: the conception and design of the study, data analysis and interpretation, final approval of the manuscript. All authors read and approved the final manuscript.

- Acknowledgements: Not applicable

- Authors' information:

Prof. Wen-Jian Meng, M.D., Ph.D.,

Department of Gastrointestinal Surgery,

West China Hospital, Sichuan University,

No. 37, Guo Xue Xiang, Chengdu 610041, China.

Tel: +86-18980606727;

E-mail: mengwenjian@126.com

\section{References}

1. Hashiguchi Y, Muro K, Saito Y, et al. Japanese Society for Cancer of the Colon and Rectum (JSCCR) guidelines 2019 for the treatment of colorectal cancer. Int J Clin Oncol 2020; 25(1). doi:10.1007/s10147-019-01485-z. 
2. Laparoscopic Surgery Committee of the Endoscopist Branch in the Chinese Medical Doctor A, Laparoscopic Surgery Committee of Colorectal Cancer Committee of Chinese Medical Doctor A. Colorectal Surgery Group of the Surgery Branch in the Chinese Medical A. [Chinese expert consensus on the diagnosis and treatment for lateral lymph node metastasis of rectal cancer (2019 edition)]. Zhonghua Wei Chang Wai Ke Za Zhi. 2019;22(10):901-12. doi:10.3760/cma.j.issn.16710274.2019.10.001.

3. Douissard J, Meyer J, Ris F, Liot E, Morel P, Buchs NC. latrogenic ureteral injuries and their prevention in colorectal surgery: results from a nationwide survey. Colorectal disease: the official journal of the Association of Coloproctology of Great Britain Ireland. 2019;21(5):595-602. doi:10.1111/codi.14552.

4. Halabi WJ, Jafari MD, Nguyen VQ, et al. Ureteral injuries in colorectal surgery: an analysis of trends, outcomes, and risk factors over a 10-year period in the United States. Diseases of the colon rectum. 2014;57(2):179-86. doi:10.1097/DCR.0000000000000033.

5. Yang X, Gu C, Hu T, et al. Is laparoscopic selective lateral lymph node dissection for locally advanced rectal cancer after neoadjuvant chemoradiotherapy safe? ANZ journal of surgery. 2019;89(11):E492E7. doi:10.1111/ans.15449.

6. Han $\mathrm{C}-\mathrm{M}$, Tan $\mathrm{H}-\mathrm{H}$, Kay N, et al. Outcome of laparoscopic repair of ureteral injury: follow-up of twelve cases. J Minim Invasive Gynecol. 2012;19(1):68-75. doi:10.1016/j.jmig.2011.09.011.

7. Andersen $\mathrm{P}$, Andersen LM, Iversen LH. latrogenic ureteral injury in colorectal cancer surgery: a nationwide study comparing laparoscopic and open approaches. Surgical endoscopy. 2015;29(6):1406-12. doi:10.1007/s00464-014-3814-1.

8. Lobo N, Kulkarni M, Hughes S, Nair R, Khan MS, Thurairaja R. Urologic Complications Following Pelvic Radiotherapy. Urology. 2018;122:1-9. doi:10.1016/j.urology.2018.07.017.

9. Gellrich J, Hakenberg OW, Oehlschläger S, Wirth MP. Manifestation, latency and management of late urological complications after curative radiotherapy for cervical carcinoma. Onkologie. 2003;26(4):334-40.

10. Chikazawa K, Imai K, Ito T, Hayashi S, Kuwata T, Konno R. Delayed Ureteral Leak Related to Use of Suspension Tapes during Laparoscopic Radical Hysterectomy. J Minim Invasive Gynecol. 2021;28(2):164-5. doi:10.1016/j.jmig.2020.05.021.

\section{Figures}



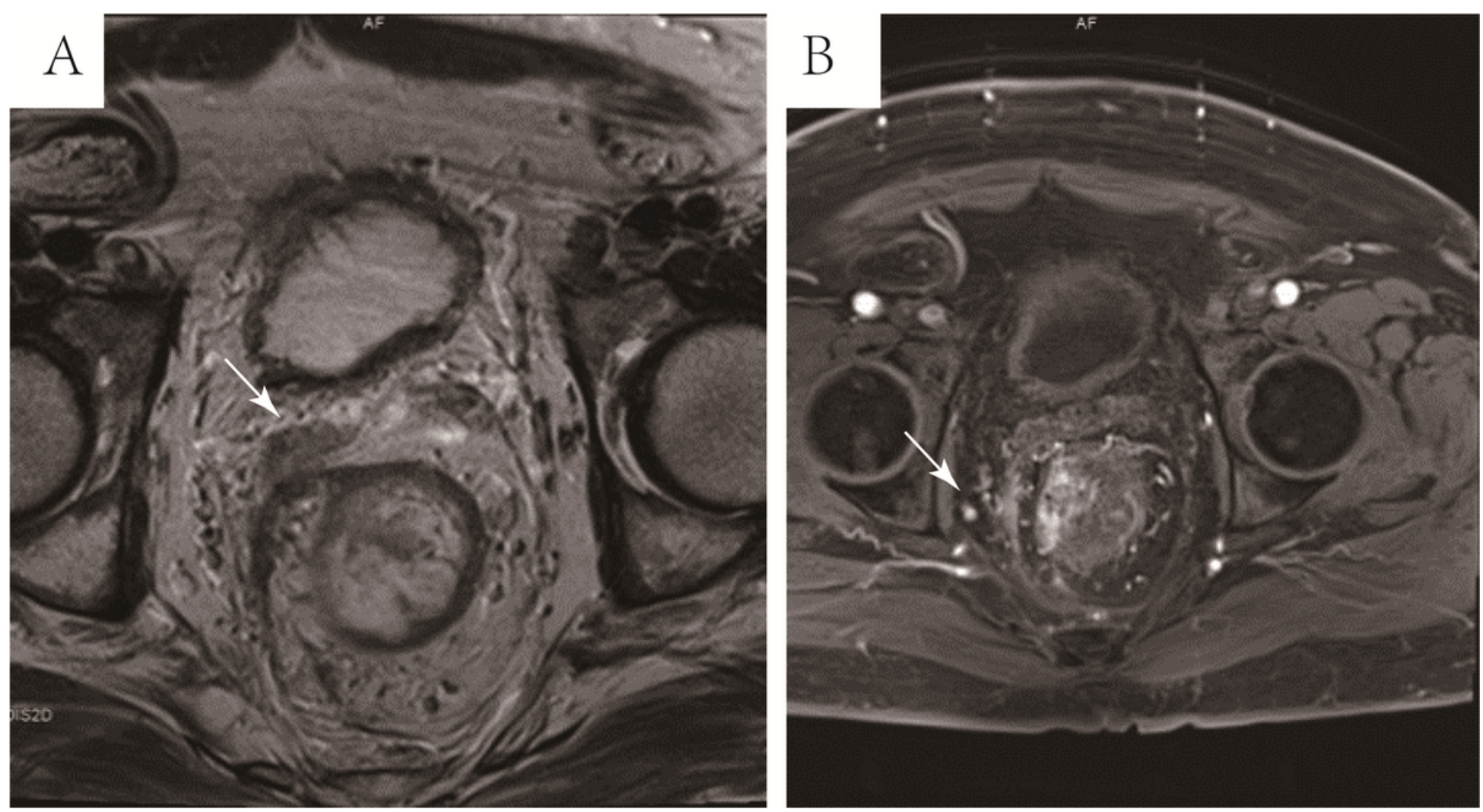

\section{Figure 1}

Seminal vesicle invasion and lateral lymph node metastasis before neoadjuvant therapy. 1A (MRI-T2, trafov): The right seminal vesicle was suspected to be invaded as shown by arrow; 1B (MRI-T1, vibe-fs): a clearly enlarged lateral lymph node on the right side can be seen.

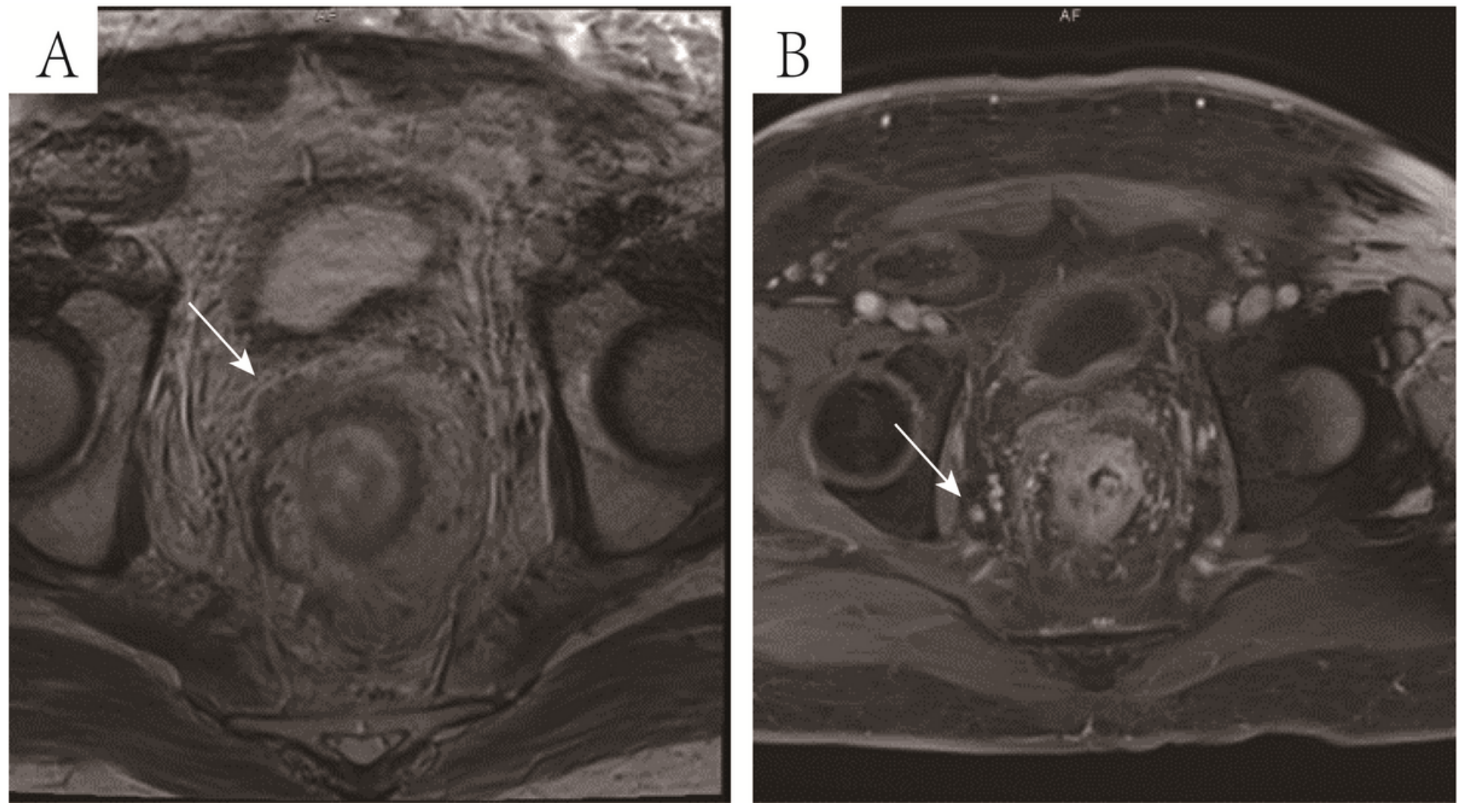




\section{Figure 2}

Seminal vesicle invasion and lateral lymph node metastasis after neoadjuvant therapy. 2A(MRI-T2, trafov): Right seminal vesicle was still invaded after neoadjuvant therapy as shown by arrow; 2B(MRIT1,vibe-fs): The enlarged lateral lymph node on the right side was still presented after neoadjuvant therapy as shown by arrow.
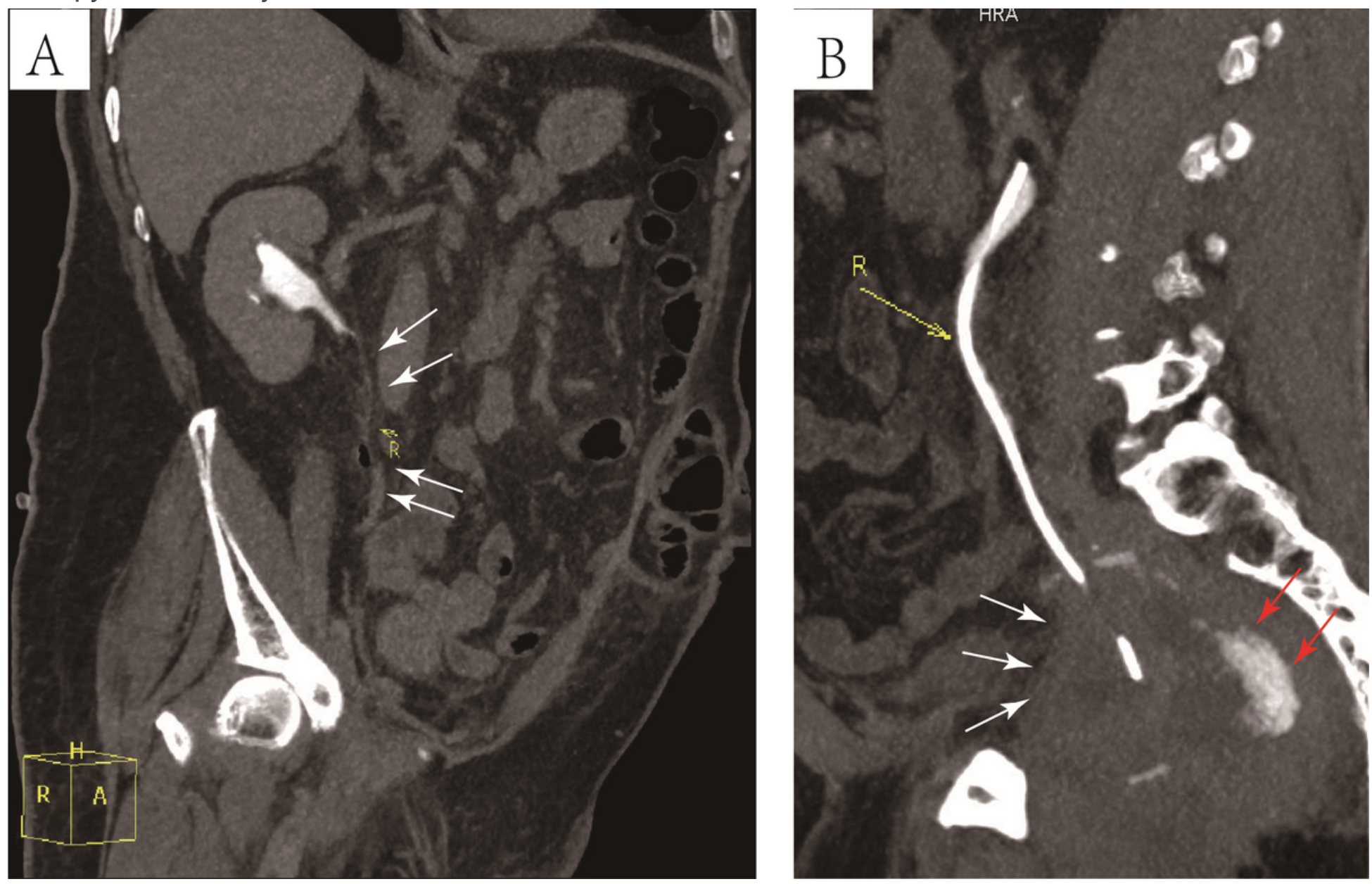

\section{Figure 3}

Sagittal view of computed tomography urography. 3A: The pelvic segment of the right ureter is not clearly displayed (arrow); 3B: A pelvic mass (white arrow) and extravasation of contrast media from the ureteral fistula (red arrow). 

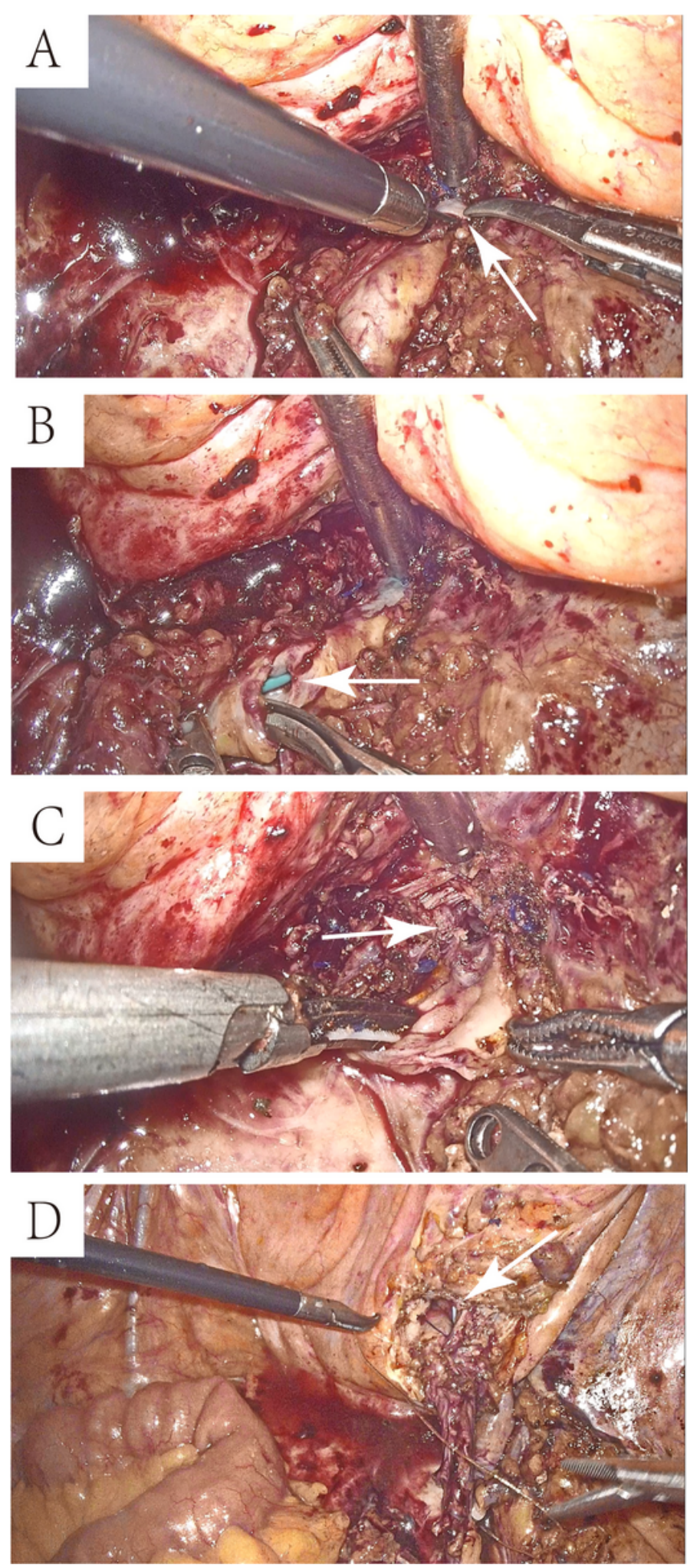

Figure 4

Laparoscopic findings and ureteral reimplantation. 4A: Distal right ureter cannot be separated from right abdominal wall and bladder wall because of severe adhesion (arrow); 4B: The ureter was cut off at the dense segment of ureteral adhesions, and a ureteral stent was observed (arrow); 4C: Dissection of the distal ureter showed inflammatory stricture of the ureter (arrow); 4D: The ureteral reimplatation was performed with a continuous suture of barbed suture with the double-J stent replacement (arrow). 


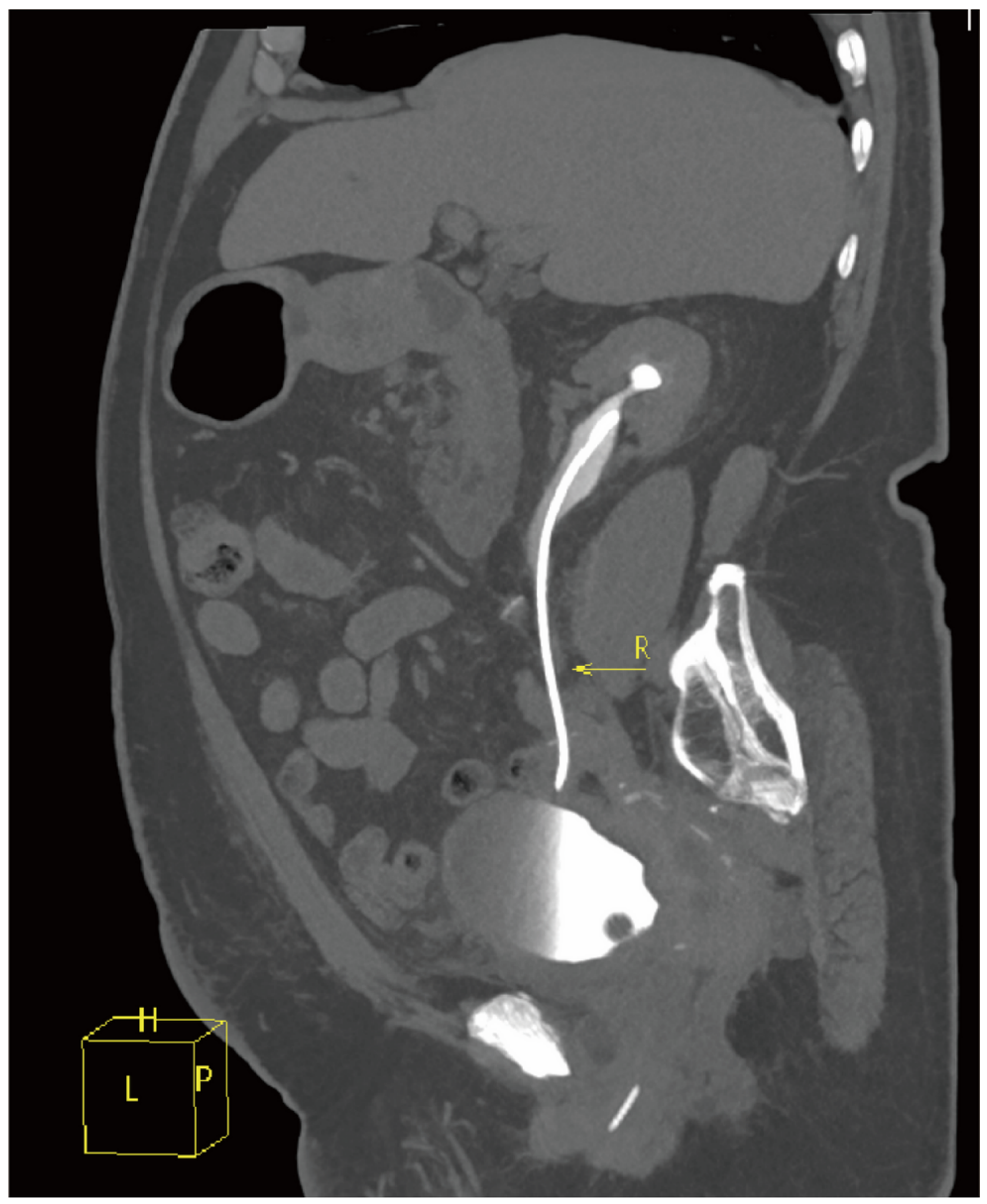

Figure 5

Enhanced ureteral CT during the follow-up period. 